\title{
Decline of FOXN1 gene expression in human thymus correlates with age: possible epigenetic regulation
}

\author{
Maria Danielma dos Santos Reis ${ }^{1,2}$, Krisztian Csomos $^{3}$, Luciene Paschoal Braga Dias ${ }^{1}$, Zsolt Prodan ${ }^{4}$, \\ Tamas Szerafin ${ }^{5}$, Wilson Savino ${ }^{1}$ and Laszlo Takacs ${ }^{2,6^{*}}$
}

\begin{abstract}
Background: Thymic involution is thought to be an important factor of age related immunodeficiency. Understanding the molecular mechanisms of human thymic senescence may lead to the discovery of novel therapeutic approaches aimed at the reestablishment of central and peripheral $T$ cell repertoire.

Results: As an initial approach, here we report that the decline of human thymic FOXN1 transcription correlates with age, while other genes, DLL1, DLL4 and WNT4, essential for thymopoiesis, are constitutively transcribed. Using a human thymic epithelial cell line (hTEC), we show that FOXN1 expression is refractory to signals that induce FOXN1 transcription in primary 3D culture conditions and by stimulation of the canonical WNT signaling pathway. Blockage of FOXN1 induceability in the hTEC line may be mediated by an epigenetic mechanism, the CpG methylation of the FOXN1 gene.

Conclusion: We showed a suppression of FOXN1 transcription both in cultured human thymic epithelial cells and in the aging thymus. We hypothesize that the underlying mechanism may be associated with changes of the DNA methylation state of the FOXN1 gene.
\end{abstract}

\section{Background}

In human, the thymus-derived naïve $\mathrm{T}$ cell repertoire, capable to exert effective protection to foreign antigens, is established during early embryonic life and it reaches maximal size in childhood [1,2], subsequently, as antigen specific $\mathrm{T}$ cells are generated, the naïve $\mathrm{T}$ cell pool is gradually depleted. Thus, the limited naïve T-cell repertoire in elderly individuals is a major contributor to age-related immunodeficiency, a frequent cause of death $[3,4]$. The immune compromised status results in the lack of effective immune response against pathogenic microrganisms and malignant cells. Because age related immunodeficiency is often life limiting as the cause of frequent nosocomial infections of the elderly, and because current treatment is insufficient, moreover it represents a significant medico-economic burden [5],

\footnotetext{
* Correspondence: TakacsL@med.unideb.hu

${ }^{2}$ Department of Human Genetics, Faculty of Medicine, University of Debrecen, Nagyerdei krt. 98, H-4032 Debrecen, Hungary

${ }^{6}$ Biosystems International Kft, Debrecen, Hungary

Full list of author information is available at the end of the article
}

there is a strong interest to develop effective and economically sound therapies. One possible strategy is the restoration of the naïve $\mathrm{T}$ cell repertoire via therapeutic regeneration of thymic activity.

Bone marrow derived stem cells migrate to the thymus where they proliferate and differentiate to $\mathrm{T}$ cell receptor (TCR) expressing $\mathrm{T}$ cells while their progeny centripetally migrate in, and eventually exit the organ. Accordingly, the recent emigrant naïve $\mathrm{T}$ cells permanently contribute to the peripheral $\mathrm{T}$ cell to maintain $\mathrm{TCR}$ repertoire diversity, and, at least in part, age related immunodeficiency is the result of the decline of naive emigrant $\mathrm{T}$ cell production [6]. Intrathymic $\mathrm{T}$ cell development is orchestrated by the microenvironment, a meshwork composed of stromal cells, such as dendritic cells, fibroblasts, macrophages and thymic epithelial cells (TEC), as well as by the extracellular matrix (ECM) molecules, which provide a unique three-dimensional environment [7]. The thymic stromal cells are distributed within the thymic epithelial space, which is divided into two main compartments, cortex and medulla [8,9]. In 
the cortical and medullary microenvironments, TEC interact with developing thymocytes via cell surface receptors, the production of ECM molecules, cytokines, chemokines and growth factors [10]. Thymic epithelial cells express (i) notch ligands which direct and restrict the bone marrow precursors to the $\mathrm{T}$ cell differentiation program [11] and (ii) self-antigen filled major histocompatibilty complex molecules (MHC) which serve as substrates for TCR repertoire selection [12].

In humans, as the thymus ages, thymic epithelial mesh is gradually replaced by adipose tissue. The process is thought to start at the first year of life and continues during aging $[8,13]$, being accompanied by a decreasing export of naive $\mathrm{T}$ cells [14]. The underlying molecular mechanisms responsible for the impairment of thymopoiesis in the aging thymus remains unclear. One possibility is that intrinsic mechanisms related to TEC physiology are impaired in old individuals, since bone marrow precursors from old animals are able to colonize the thymus [15]. In fact, some studies showed that TEC proliferation is lower in old animals and it was also demonstrated that aging mice have higher percentage of apoptotic and senescent TECs $[15,16]$.

Studies in rodent models pointed out that the transcriptional factor forkhead box protein N1 (FOXN1) is both necessary and seemingly sufficient to induce differentiation of functional TEC [17, 18]. FOXN1 appears on day 11 during mouse embryonic development, the sixth week of gestation in humans, and induces the thymic organogenesis program presumably under the control of WNT family of glycoproteins, namely, by WNT-4 $[2,19,20]$. In a model with inducible Cre mediated deletion of an SV40 driven transgenic hypomorphic Foxn1 allele, it has been demonstrated, that FOXN1 in TEC induces the expression of MHC II, CD40, PAX1, cathepsin-L, the chemokine CCL25 and the NOTCH ligand Delta-like 4 (DLL4), thus highlighting its orchestrating role in T cell maturation [21]. The lack of FOXN1 in mice and rats results in the absence or the incomplete development of TEC and the thymic epithelial mesh, combined with severe immunodeficiency known as the nude phenotype [22]. Nude mice carry a single base pair deletion at exon 3 of the Foxn1 gene, which results in aberrant protein production, lacking the DNA-binding and the transcription activation domains, necessary for FOXN1 protein function [23, 24]. Similar phenotype was found in human, carrying a rare non-sense mutation at the residue 255 of the FOXN1 protein, resulting from a single base substitution in exon 5 of the FOXN1 gene [25]. Recently, Bredenkamp and co-workers showed that mouse embryonic fibroblasts transfected with inducible Foxn1 transdifferentiated to functional TECs upon induction [18]. These Foxn1 induced TECs support T cell development in vitro and in vivo. The data clearly demonstrate the central role of FOXN1 in thymic epithelial function and organogenesis. Murine models showed a decrease on Foxn 1 expression in aged thymus [26, 27]. Others found that the decline of Foxn1 expression results in a decrease of thymus cellularity and function, compared to normal aged murine thymus $[28,29]$. In contrast, thymus from aged mice, with high transgenic Foxn1 levels, presented morphology and T cell maturation similar to the thymus of young mice [30]. In addition, it was demonstrated that induction on Foxn1 expression in a murine aging model was able to restore thymic architecture and $\mathrm{T}$ cell export, similar to preinvoluted thymus [31]. Despite the overwhelming genetic [32] and molecular evidence pointing to the FOXN1 gene as the key regulator of thymopoiesis is aging, little is known on human FOXN1 function and its regulation.

As an initial approach, from consented, young and aging donors thymic biopsy specimens and a human postnatal TEC line (hTEC) [33], we tested the expression of genes that have been reported to be essential for T cell development, particularly the inducibility of the FOXN1 gene expression. Subsequently, we tested the methylation status of predicted transcriptional regulatory regions of the human FOXN1 gene inthe cultured human TEC line.

\section{Results}

\section{Expression of DLL1, DLL4, FOXN1 and WNT-4 genes in} human thymic samples

Thymic samples were divided into three different groups according the donor's age. The groups were created by considering major hormonal transitions as shown previously [34]. The "Postnatal" group comprises samples from 5 days-old to 1 year-old (a total of four samples); the "Child-adolescent" group contains samples whose donors are 7, 10, 14 and 17 years-old (a total of four samples); the "Adult" group included samples from donors, 49, 57, 59, 66, 75 and 78 years-old (seven samples total). As represented in Fig. 1a and b, respectively, the Delta-like 1 (DLL1) and 4 (DLL4) genes presented low relative levels in the younger groups, showing a significant elevation in the "Adult" group, while the Forkhead box protein N1 (FOXN1) gene expression was significantly decreasing from "Postnatal" through "Child-adolescent" to the "Adult" group (Fig. 1C). It is important to note that age vs. normalized expression levels of FOXN1 do correlate significantly (see Additional file 1: Figure S1). The wingless-type MMTV integration site family, member 4 (WNT-4) gene expression seemed constitutive, not showing remarkable differences over time (Fig. 1d).

\section{Histology, thymic epithelial organization and FOXN1 protein expression}

The "Postnatal" thymic samples, from 5 days-old to 1 yearold, showed typical morphology, with the surrounding connective capsule, trabeculae, cortical and medullary regions. 


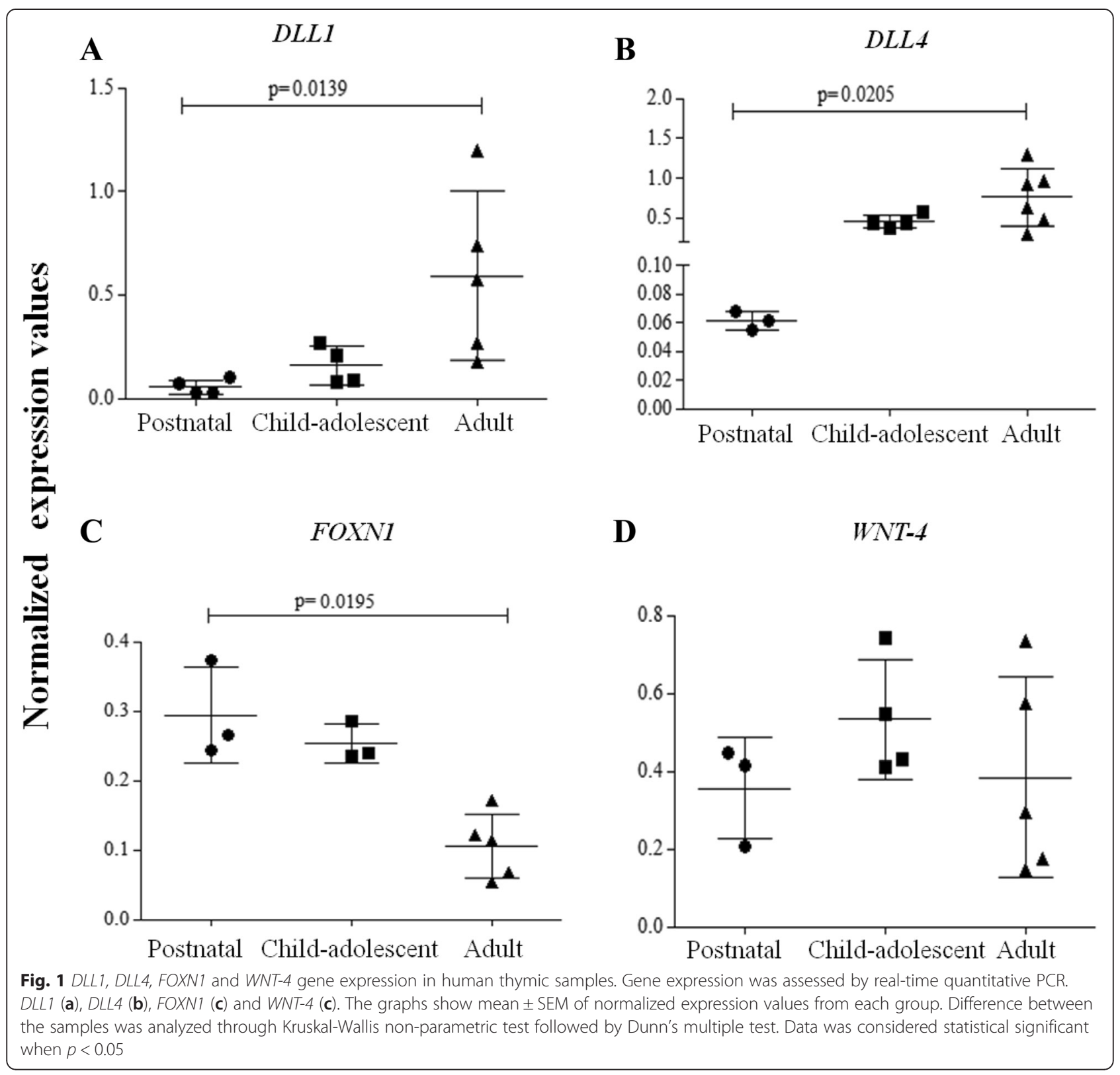

With the exception of occasional individual fat cells in the interlobular areas, we did not detect thymic adipose tissue in these samples (Fig. 2a). Cytokeratin labelling pattern revealed the typical thin mesh distributed throughout the thymic cortex, and with more dense medullary TEC (Fig. 2c). In the "Child-adolescent" group we detected considerable amount of organized adipose tissue embedded in interlobular areas (See Additional file 1: Figure S2). It is important to note that the cortex to medulla ratio is lower, thus the thymic lobes seem to possess relatively larger medullary areas and overall, smaller lobes (See Additional file 1: Figure S2). In the "Adult" group, intrathymic adipose tissue dominates, and no well-defined cortical and medullary regions could be distinguished (Fig. 2b). The cytokeratin organization is severely altered, and this becomes more profound with increasing age (Fig. 2d).

Since with qPCR we found significantly lower FOXN1 gene expression in the "Adult" group compared to the younger groups of thymic samples, we tested and compared the expression of FOXN1 protein in samples from the "Postnatal" and "Adult" groups by immunofluorescence. We observed characteristic nuclear FOXN1 staining in the "Postnatal" group. FOXN1 positive cells were distributed in the cortex, although the majority of epithelial cells were negative, suggesting heterogeneity of thymic epithelium with respect to FOXN1 expression (Fig. 2e). Surprisingly, no FOXN1 protein expression 

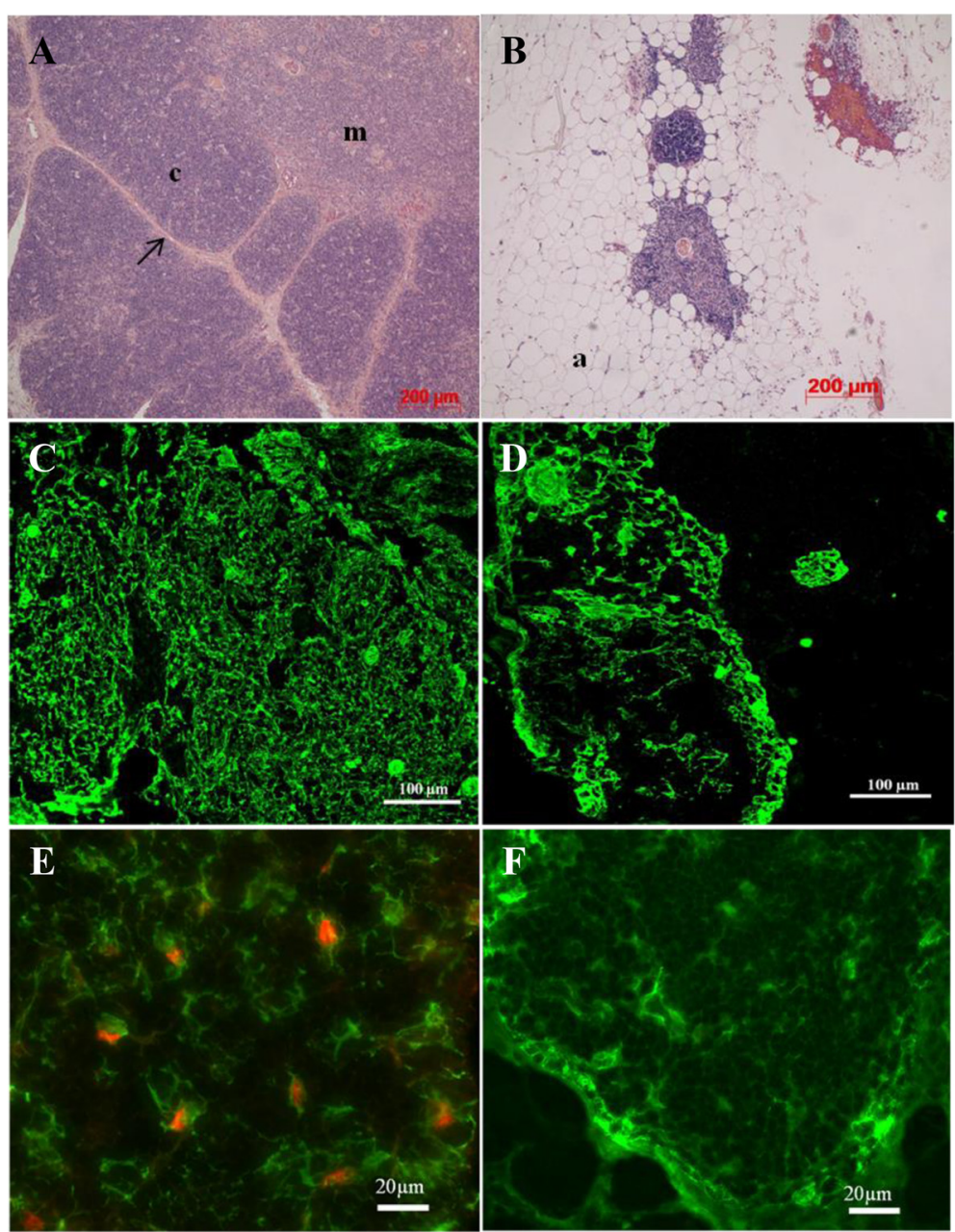

Fig. 2 Histology, thymic epithelial organization and FOXN1 protein expression. Photomicrographs show morphological aspects of thymus samples from (a) five days ("Postnatal" group) and (b) 57 years-old donors ("Adult" group). Paraffin sections were stained with haematoxilin-eosin stain and analyzed by light microscopic examination. (4x, objective magnification). $c=$ cortical region; $m=$ medullary region; $a=$ Adipose tissue; arrow = connective tissue septum. Panels (c) and (d) show cytokeratin staining of "Postnatal" (5 days-old) and "Adult" thymic samples (57 years-old), respectively. Frozen thymus sections were reacted with specific pan anti-cytokeratin polyclonal rabbit antibody followed by Alexa-488 conjugated anti-rabbit Ig antibody (green) before visualization in the fluorescent microscope. (20x, objective magnification). Photomicrographs (e) and (f) show FOXN1 protein expression in thymus cryosections from "Postnatal" (5 days-old) and "Adult" donors (57 years-old), respectively. In order to demonstrate thymic epithelium associated FOXN1 expression, the sections were first reacted with anti-human FOXN1 antibody (red) and subsequently double stained with pan anti-cytokeratin antibody (green). (20x, objective magnification)

was detectable in samples from the "Adult" group, even though the thymic epithelium was clearly detectable, typical staining patterns are shown on Fig. 2f.

\section{hTEC cell line, a model of aging thymic epithelium}

Recent reports indicate that thymocyte development is induced and supported by Foxn1 transduced mouse fibroblast cells that transdifferentiate to functional thymic epithelium [18]. Thymus derived epithelial cell lines and primary monolayers of TEC cultures do not induce full scale $\mathrm{T}$ cell development $[35,36]$, and this may be related to a decrease of FOXN1 expression $[19,37]$ by a yet unidentified mechanisms. A human TEC line (hTEC), which expresses membrane proteins specific for human TEC (in vivo), and able to adhere to $\mathrm{T}$ cell precursors, immature and mature naïve $\mathrm{T}$ cells was chosen here for further investigations [33]. In preliminary experiments, we found no FOXN1 mRNA expression in hTEC cells (Fig. 3d). Nevertheless, other tested thymic epithelium genes that are necessary for $\mathrm{T}$ cell development were expressed (Fig. 3e and f). Due to the lacking FOXN1 expression, the striking gene expression pattern similarity with the aging human thymus, the hTEC line served as a model of the aging thymic epithelium here. Using this model we tested conditions that have been reported to induce FOXN1 expression [19, 37]. First, we prepared three-dimensional 


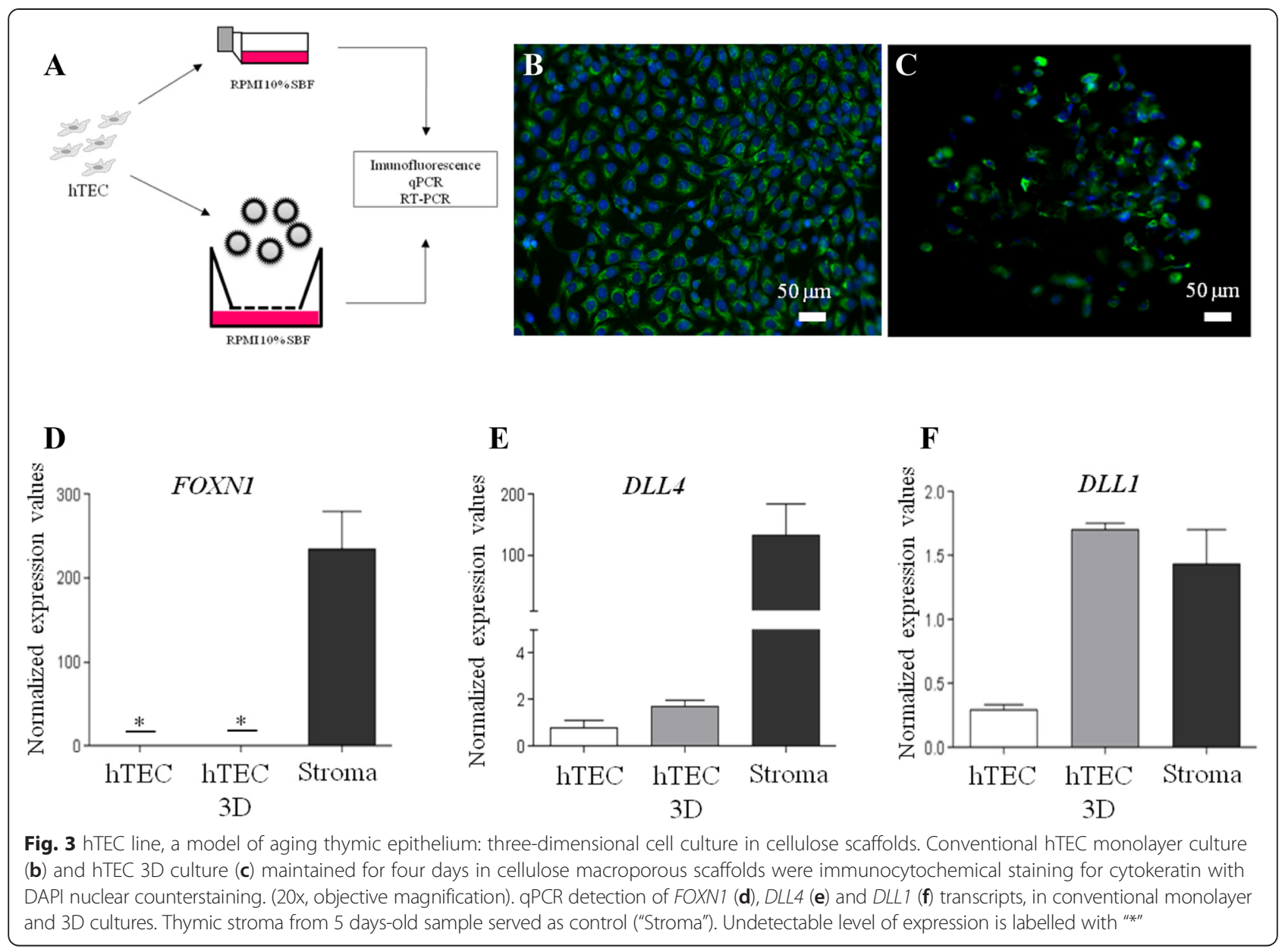

(3D) cultures of hTEC (Fig. 3a) and found that both conventional monolayer and 3D hTEC cultures expressed and maintained epithelial characteristics as ascertained by cytokeratin expression (Fig. 3a and b, respectively). However, neither conventional nor 3D cultures expressed detectable FOXN1 transcripts, despite the fact that the 3D culture system based on microspherical substrate did function, since DLL4 and DLL1 transcripts were detectable and showed remarkable induction by the 3D culture conditions (Fig. 3d, e and f). It is worth to note that we tested $\mathrm{T}$ cell development inducing/maintaining capacity of the hTEC by co-culturing thymocytes, both in monolayer and in 3D cultures, but we did not observed increased thymocyte production in the cultures (data not shown).

In thymus-derived primary epithelial cultures, lithium chloride $(\mathrm{LiCl})$, a glycogen synthase kinase- $3 \beta$ inhibitor and a mimetic of the canonical WNT-4 signaling pathway [38], was shown to induce Foxn1 transcription, which was accompanied by $\beta$-catenin translocation to the nucleus [19]. Here, we tested whether $\mathrm{LiCl}$ induced FOXN1 expression in hTEC line (Fig. 4). Upon exposure of hTEC cultures to LiCl treatment for $6 \mathrm{~h}$ and for $18 \mathrm{~h}$, characteristic morphological changes were observed, namely intensive cell spreading at $6 \mathrm{~h}$ (Fig. 4b) and overgrowth, apoptotic aggregation of cell clusters at $18 \mathrm{~h}$ (Fig. 4c), with accompanied $\beta$-catenin reorganization, which included detectable $\beta$-catenin nuclear translocation at in the $6 \mathrm{~h}$ of culture (Additional file 1: Figure S3). As $\mathrm{LiCl}$ induced the expected biological response in hTEC, we tested FOXN1expression using immunostaining and RT-PCR. FOXN1 protein expression was not detectable on untreated hTEC (Fig. 4d), in agreement with the qPCR data showed on Fig. 3c. Upon LiCl treatment, the FOXN1 gene remained silent, with no detectable FOXN1 protein in the LiCl-treated hTEC cultures (Fig. 4e and f) and no detectable transcripts produced in the control or in LiCl-treated cell cultures (Fig. 4g).

\section{FOXN1 genomic context of candidate CpG methylation substrate, C20}

As the hTEC line was refractive to FOXN1 transcriptional induction, we thought to investigate DNA methylation, a possible epigenetic silencing mechanism, in hTEC line. Based on reported DNA methylation data from Encyclopedia of DNA elements (ENCODE) consortium, we first identified several CpG methylation sites, 


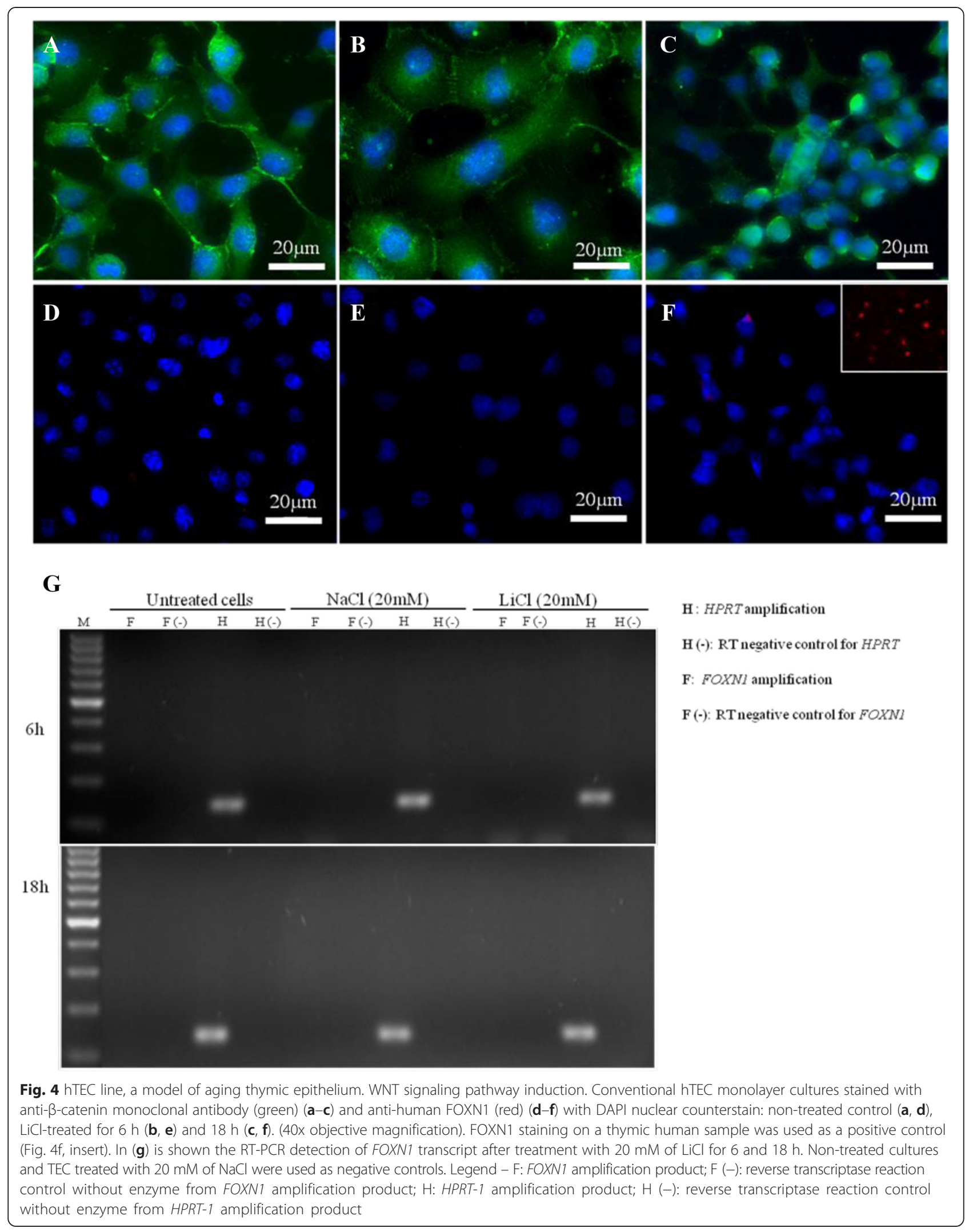


potentially involved in transcriptional regulation of the FOXN1 gene. In the data set, there are 139 candidate residues for CpG methylation within the FOXN1 gene. The candidate CpG residues are distributed in the promoter region, in exons $1,2,3,7,8,9$ and in introns 1,2 , $3,6,7$ e 8 . We chose one candidate region to test differential methylation: (i) because of overlap with FOXN1 regulatory regions, (ii) the high GC \% context, (iii) the presence of a predicted CpG island (Fig. $5 \mathrm{a}$ and b). The selected region is a $259 \mathrm{bp}$-sequence located at the first FOXN1 intron, was termed C20 (Fig. 5a). The C20 region is close to the predicted FOXN1 promoter and it satisfies all criteria for being "CpG methylation target" for gene expression regulation, such as high GC content and being within a CpG island. Specifically, the C20 sequence contains a simple repeat (CCCG) $)_{n=3}$ of $27 \mathrm{bp}$ inside a CpG island of 100 bp-s (Fig. 5b, upper panel). Experimental data from ENCODE demonstrated that C20 is located within regulatory regions defined by histone modifications and it is included in a DNA-binding site cluster for RNA polymerase and the zinc-finger protein CTCF (CCCTC-binding factor) (Fig. 5b). There are 13 potential $\mathrm{CpG}$ methylation sites in this genomic sequence (Fig. 5b, low panel). Remarkably, as deduced from the ENCODE database [39], 8 of the 13 CpG cytosine residues show differential methylation pattern with respect to FOXN1 expressing human skin $[25,40]$ and FOXN1 non-expressing human leukocytes [41] as shown on Fig. 5c and d, respectively.

\section{FOXN1/C20 region CpG methylation status of hTEC line}

We tested CpG methylation status of the 13 cytosine nucleotides present in the $\mathrm{C} 20$ sequence by bisulfite conversion, followed by PCR fragment cloning and DNA sequencing (See Additional file 1: Figure S4). Methylation status deduced from sequence analysis of 10 independent clones of the $\mathrm{C} 20$ region of hTEC cell line resembles of the FOXN1 non-expressing skin, suggesting that at least in the case of hTEC (Fig. 5e and f), CpG methylation within the $\mathrm{C} 20$ region correlates with the observed lack of expression and resistance to induction.

\section{Discussion}

Immunosenescence is a physiological process characterized by the decline of both adaptive and innate immune functions [42]. The decreased ability to respond to antigens, at least in human, is thought to be the result of decreasing naïve $\mathrm{T}$ cell export that ultimately limits peripheral $\mathrm{T}$ cell repertoire in adults [43]. However, maintenance of naïve $\mathrm{T}$ cell repertoire in the periphery is thought to involve homeostatic regulation of proliferation [14, 44, 45]. Naïve T-cells derive from the thymus, however, histological signs of thymic involution are evident already at the first year of life [8]. As the organ ages, the thymic interstitium and ultimately intrathymic TEC areas are infiltrated by adipose tissue and peripheral lymphoid cells resulting in decreased thymopoiesis, eventually limiting the peripheral naïve $\mathrm{T}$ cell repertoire [16].

Little is known about the molecular mechanisms underlying thymic involution. Current reviews in the literature disagree whether thymic involution is organ autonomous, or is it due exclusively to decreasing function of TECs, or, and in addition, whether it involves changes in bone marrow derived stem cell activity and migration $[14,46]$ ?

To address molecular mechanisms involved in human thymic involution during aging, first we tested gene expression levels of candidate genes in thymic biopsy specimen from days-old 5 to 78 years-old $(n=15)$ from consented cardiac surgery patients and in a human TEC line (hTEC). We choose DLL1, DLL4, FOXN1 and WNT4 genes for the analysis as these genes have been reported to be necessary for inducing intrathymic $\mathrm{T}$ cell development in mouse models [17, 36, 47, 48]. Particularly, FOXN1 was selected because of reports indicating reduced expression in the ageing mouse thymus [26] and because recently, Bredenkamp and co-workers (2014) [18] showed that mouse embryonic fibroblasts transfected with inducible mouse Foxn1 support $\mathrm{T}$ cell development in vitro and in vivo. Moreover, critical pathways for thymic development involve $\mathrm{NOTCH}$ ligands and receptors. In mouse models it was demonstrated that DLL1, DLL4, Jagged-1 (JAG-1) e JAG-2 ligands are expressed on TEC with their receptors expressed on thymocytes [49-51]. It is well established that DLL4 produced by TEC is responsible for $\mathrm{T}$ cell lineage commitment, being necessary for the maintenance of the three dimensional architecture of the thymic microenvironment $[37,47,52]$. Despite the broad range of studies on NOTCH and its ligands, little is known about their participation in thymic involution, especially in the human thymus. In addition to the NOTCH family of ligands, we also evaluated WNT-4 gene expression. WNT-4 glycoprotein is secreted and it is produced both by TEC and thymocytes [53]. In a mouse model, WNT-4 was shown to play important role in organ development, $\mathrm{T}$ lymphocyte differentiation, and it was also associated with thymic involution $[27,48,54,55]$. The WNT-4 glycoprotein also operates on TEC through induction of Foxn1 expression in the mouse [19]. The decrease in the expression of FOXN1 has been studied as a hallmark of thymic senescence, although nothing is known on its expression in the human thymus during aging.

Our quantitative PCR gene expression experiments indicate that $D L L 1$ and $D L L 4$ are constitutively expressed in the human thymus, with a significant increase in the "Adult" group of thymus donors. In contrast, studies in experimental mouse models showed decrease in expression of Dll4 in cortical TEC from old animals [31]. Since 


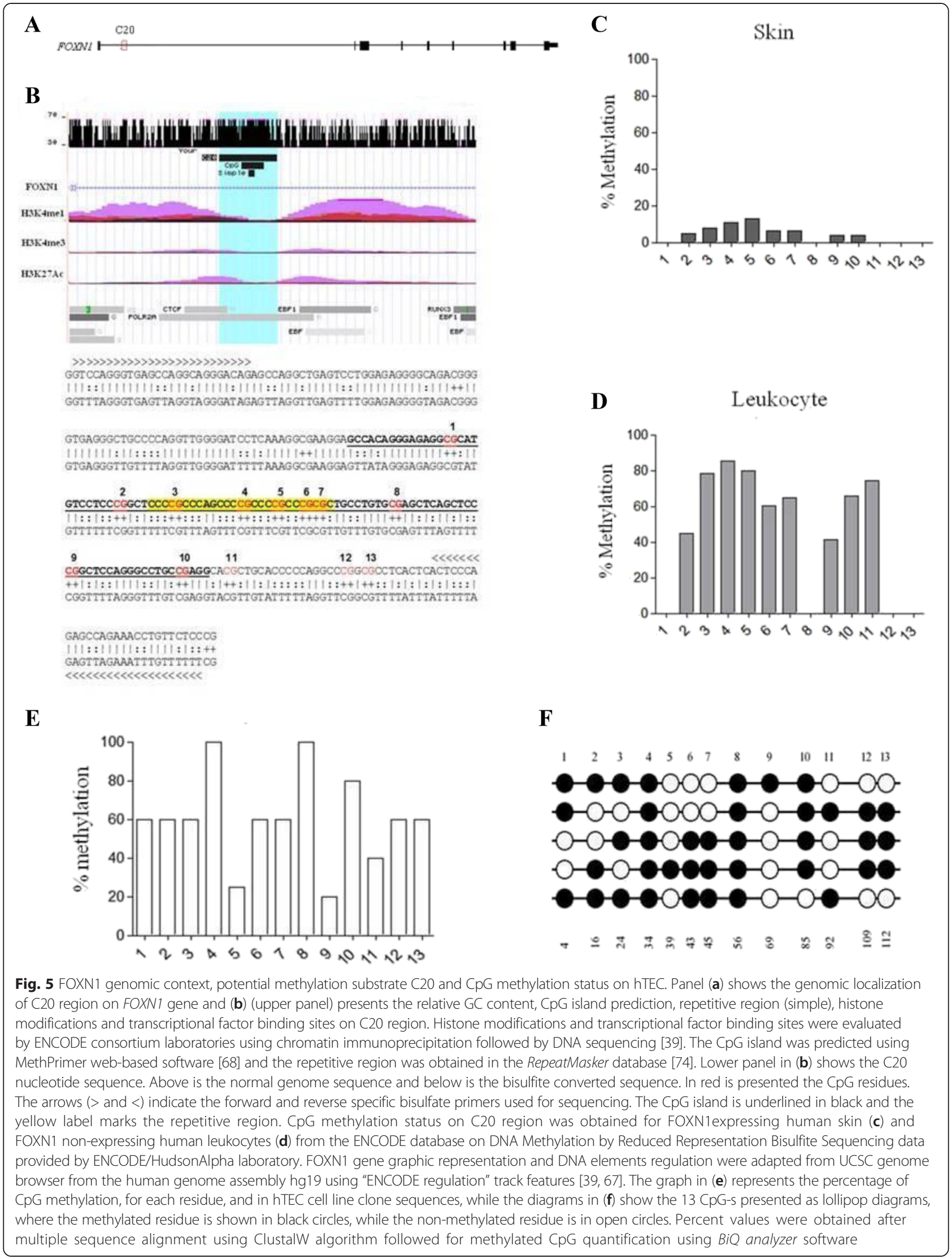


TEC isolation in our samples was not possible, it is reasonable to think that the high expression of DLL1 and DLL4 observed in "Adult" group is related to the presence of adipocytes in these samples. In fact, DLL4 expression has been reported in adipose tissue from human and animals subjected to high-fat diet [56]. Nevertheless, due to their increasing expression, DLL1 and DLL4 are not likely to limit human thymopoesis. Similarly to the NOTCH ligands, WNT-4 gene is transcriptionally active in the human thymus in all tested groups, with a slight increase in expression in the "Child-adolescent" thymus samples. Although gene expression studies from whole organ are not suitable to address cell type specific expression, we suggest that the soluble factor, WNT-4, may be present in the aging thymus, thus it is not likely to limit thymopoetic activity either.

For the first time, we report here a striking three-fold decrease of FOXN1 expression over time in the human thymus, when comparing the "Postnatal" group with the "Adult" group. In fact, the decrease of TEC associated expression levels may be markedly higher, as due to the relatively lower lymphocyte content of the aging thymus, the relative abundance of TEC is increasing. We thus suggest that FOXN1 expression may limit thymopoiesis and its reduced expression may be responsible for thymic senescence. This notion is strongly supported by the fact that, although TECs are present in the samples of typical morphologically-defined aging thymus, FOXN1 protein were not detected in epithelial cells. The notion is also inline with recent reports indicating that inducible Foxn1 expression in mouse embryonic fibroblasts mediates trans-differentiation to functional TEC [18], suggesting that, at least in mice, FOXN1 is necessary and seemingly sufficient to induce thymopoiesis. Key role of FOXN1 gene in human thymopoiesis is likely as the phenotype of the FOXN1 null mutant humans $[25,57]$ is very similar to that of the nude phenotype of homozygous Foxn1 mutant mice and rats [22, 24]. Moreover, thymic histology of Foxn1 homozygous mutants strongly resembles that of thymic samples from aging individuals $[28,29]$.

To model age-related changes, we tested a human thymus derived epithelial cell line, hTEC, for expression of FOXN1, DLL1 and DLL4 genes. Although, the hTEC does bind to early thymocyte subsets, it does not induce or support full scale $\mathrm{T}$ cell development [33]. As we found no detectable FOXN1 expression in cultured hTEC (while DLL1 and DLL4 gene expression were definitely detected) we tested whether hTEC cells would respond to stimuli reported to induce FOXN1 expression. To this end, we used 3D culture conditions shown to induce FOXN1 expression in hTEC cultures [37] and $\mathrm{LiCl}$ treatment, which was also reported to induce downstream canonic pathway of WNT signaling, including Foxn1 expression induction in murine TEC [19]. The
hTEC cell line did not respond to the tested specific stimulatory signals by increasing FOXN1 expression. We therefore suggest that although WNT-4 may regulate FOXN1 expression, it requires FOXN1 gene to be responsive for inductive signaling by WNT-4. Thus hTEC is seemingly resistant to FOXN1-inducing stimuli. Among others, such resistance could be the result of transcriptional silencing, mediated by epigenetic regulatory mechanisms.

One of the most important epigenetic mechanisms that is often involved in transcriptional regulation during development is CpG methylation [58]. To investigate the methylation status of CpG residues in the FOXN1 gene in the hTEC, we tested a candidate regulatory region (we named C20) by bisulfite conversion followed by DNA sequencing. In the $\mathrm{C} 20$ candidate region FOXN1 expressing skin cells $[25,39,40]$ show minimal methylation in 8 of the 13 candidate CpGs of the C20 region, while FOXN1 non-expressing leukocyte $[39,41]$ is highly methylated. We could observe that hTEC line DNA exhibits remarkably elevated methylation compared to skin DNA (from the ENCODE database). Overall methylation is decreasing with age, and we have detected this in the human thymic biopsy samples (ongoing studies; see Additional file 1: Figure S5) consistently with the age-dependent demethylation of CpG-s that has been reported in many tissues [59-61]. Hypermethylation of the C20 segment of the hTEC provides a strong clue supporting our hypothesis, namely that hypermethylation may gradually silence the FOXN1 ultimately leading to decreased thymopoesis.

\section{Conclusions}

For the first time in human thymus we show that FOXN1 expression decreases with age. Considering the central role of FOXN1 in thymopoesis we suggest that mechanism affecting FOXN1 expression regulation may be critically involved in thymic senescence. To support the notion we show that FOXN1 gene in hTEC line is resistant to transcriptional induction that mimic physiological conditions. With respect to the hTEC line, our data supports the hypothesis that resistance of the FOXN1 gene to physiological and chemical stimulatory signals may be mediated by an epigenetic mechanism, namely CpG methylation of specific regulatory regions(s) like $\mathrm{C} 20$. Although, the depth of the experiments and the heterogeneous tissue are severely limiting our conclusions, as a working hypothesis for future experimentation, we suggest that developmentally regulated $\mathrm{CpG}$ methylation of FOXN1 may be a critical molecular mechanism behind thymic senescence and age related immunodeficiency. As our hypothesis needs further support from more accessible and specific rodent and in-vitro models, we plan to continue towards further understanding of epigenetic 
mechanisms behind thymic senescence with the hope that we may open avenues for therapeutic modulation via small molecule drugs.

\section{Methods}

\section{Cell culture}

The human TEC line (hTEC) was originally obtained from an infant thymus by primary explant culture and limiting dilution cloning [33], however since its derivation the cell lines has been passaged over 100 times. It has been kindly provided by Dr. Maria Luiza Toribio (Universidad Autonoma de Madrid, Madrid, Spain). These cells were shown to express cytokeratins and several surface proteins, such as CD71, CD40, MHC I (HLA-ABC), MHC II (HLA-DR) ${ }^{\text {low }}$, ICAM-1, LFA-3, CD44, integrin-type ECM receptors including VLA-4, VLA-5 and VLA-6 $[32,62]$. Cells were cultured in $10 \%$ fetal calf serum (Cultilab, São Paulo, Brazil) supplemented RPMI 1640 medium (Mediatech, Virginia, USA) at $37{ }^{\circ} \mathrm{C}$ in a $5 \% \mathrm{CO}_{2}$ atmosphere. Alternatively, these cells were cultured in three-dimensional cell culture system using macroporous cellulose microcarriers (Cytopore ${ }^{\mathrm{Tx}}$, Asaki Kasei Medical Co., GE Healthcare, Japan) as described previously [63] with some modifications. Briefly, $100 \mu \mathrm{L}$ of phosphate-buffered saline (PBS)-hydrated macroporous cellulose microcarriers were colonized with cells in a volume of the $2 \times 10^{5}$ cells under nitrocellulose membrane from Transwell $^{\mathrm{Tm}}$ inserts (Costar, Corning Incorporated, USA) and cultured for 4 days with complete medium at $37{ }^{\circ} \mathrm{C}$ in a $5 \% \mathrm{CO}_{2}$ atmosphere. Under these conditions, the cells were allowed to grow in 3D environment (Fig. 3a).

\section{Sample collection and histology}

Thymus samples were obtained from consented paediatric and adult patients undergoing cardiac surgery at the György Gottsegen National Institute for Cardiology, (Semmelweis University, Budapest, Hungary) and from the Department of Cardiac Surgery of the Clinical Center, University of Debrecen (Debrecen, Hungary) under a protocol approved by the Hungarian Ethics Committee for Science and Research (11739-/2014/EKU 107/2014.) and the local institutional review boards. Patients' blood counts were in the normal range in all cases as this is prerequisite condition for selected eletive surgeries. Fresh thymus fragments were cleaned, washed in PBS and fixed in neutral buffered formalin for routine histological examination. Additional $3 \times 3 \times 3 \mathrm{~mm}$ fragments were snap frozen and kept at $-80^{\circ} \mathrm{C}$ until use.

\section{Lithium chloride treatment}

hTEC line were cultured under standard conditions with complete medium for two days, and then treated with $20 \mathrm{mM}$ of lithium chloride ( $\mathrm{LiCl}$ ) for 6 and $18 \mathrm{~h}$ as previously described to induce the WNT signaling pathway
$[19,64]$. Non-treated cells and sodium chloride $(\mathrm{NaCl})-$ treated cells were used as negative controls. After treatment, cells were used for RNA isolation or fixed with $100 \%$ methanol for imunofluorescence staining.

\section{Immunofluorescence}

Immunofluorescence assays were used to evaluate FOXN1, cytokeratin and $\beta$-catenin protein expression in thymus samples and hTEC line cultured under various conditions. Briefly, thymic frozen sections fixed in acetone, and hTEC cultures fixed with $100 \%$ methanol, were re-hydrated in PBS and incubated for $30 \mathrm{~min}$ with PBS containing $1 \%$ bovine serum albumin to block unspecific binding. Next, the specimens were incubated with primary antibodies specific for human FOXN1 (donkey IgG, 1:50; Santa Cruz Biotechnology, Heidelberg, Germany), pan-cytokeratin (rabbit IgG, 1:100; DAKO, Aligent Technologies, Glostrup, Denmark) or human $\beta$-catenin (mouse monoclonal antibody, $\operatorname{IgG}_{1}$, 1:30; Santa Cruz Biotechnology, Heidelberg, Germany) for $1 \mathrm{~h}$ and $30 \mathrm{~min}$ at room temperature in a humidified chamber. After washings with PBS, the slides were incubated with corresponding fluorochrome-labeled secondary antibody for $45 \mathrm{~min}$ at room temperature, in a dark humidified chamber. Secondary reagents were: goat antirabbit (1:400; Molecular Probes, Life Technologies), goat anti-mouse (1:400; Molecular Probes, Life Technologies) and donkey anti-goat (1:400; Invitrogen). Next, after washing in PBS, the specimens were mounted for examination in the fluorescence microscope, a Zeiss Axio Imager A2 (Carl Zeiss, Oberkochen, Germany) equipped with Axio Vision Release 4.8.2 software (Zeiss). Negative controls in which primary antibodies were replaced by unrelated immunoglobulins or in which the secondary antibody was used alone did not generate significant immunolabeling.

\section{RNA isolation, CDNA synthesis and PCR assays}

Total RNA from thymus samples and hTEC line were purified by phenol-chloroform and used as templates for first strand cDNA synthesis followed by gene expression analysis via RT-PCR and quantitative PCR assays as follows. Small fragments of frozen thymi were homogenised with tissue homogenizer (T10 Basic, IKA ${ }^{\circ}$ ) in $1 \mathrm{ml}$ of Trizol (TriReagent ${ }^{\circ}$, Molecular Research Center, Ohio, USA) whereas hTEC cultured in conventional monolayers or in 3D scaffolds were released from the substrate with trypsin/EDTA treatment for $10 \mathrm{~min}$, centrifuged, washed in PBS and homogenized with $1 \mathrm{ml}$ of Trizol (TriReagent ${ }^{\oplus}$, Molecular Research Center, Ohio, USA). After homogenization, RNA was purified according manufacturer's instructions. Total RNA concentration and purity were determined by the ratio of absorbance readings at 260 vs. $280 \mathrm{~nm}$ on NanoDrop ND 
2000 spectrometer (Thermo Scientific, Delaware, USA). Up to 2 micrograms of total RNA were used to synthesize cDNA using high capacity cDNA reverse transcription kit (Applied Biosystems, California, USA) following manufacturer's protocol. Quantitative PCR and RT-PCR reactions were performed using oligonucleotides previously described in the literature or specifically designed based on the target gene sequences reported in NCBI GenBank using the Primer3 software from the NCBI/BLAST platform [65]. Specific sequences are shown in Table 1. For RT-PCR reactions, eight microliters of diluted cDNA (1:5) were mixed with nuclease-free water, 1.5 millimolar of $\mathrm{MgCl}_{2}$, 1X Taq polimerase buffer, 0.025 millimolar of dNTPs, 0.05 units per microliter of Taq polimerase and 0.5 micromolar of each primer in a $50 \mu$ l of total volume (all from Applied Biosystems, California, USA except the nuclease-free water and primers). The cycling conditions were as follows: initial denaturation at $94{ }^{\circ} \mathrm{C}$ for $2 \mathrm{~min}$, followed by 30 cycles with denaturation $94{ }^{\circ} \mathrm{C}$ for $30 \mathrm{~s}$, annealing at $60{ }^{\circ} \mathrm{C}$ for $30 \mathrm{~s}$, and elongation at $72{ }^{\circ} \mathrm{C}$ for $30 \mathrm{~s}$ using the A\&B thermocycler 2720 (Applied Biosystems, California, USA). Ten microliters from each PCR reaction were run on $2 \%$ agarose gels at $90 \mathrm{~V}$. The PCR products were visualized under UV light using ethidium bromide staining. The quantitative PCR reactions were performed using $5 \mu \mathrm{l}$ from 5 -fold diluted cDNA sample mixed with Maxima SYBR Green/ROX qPCR Master Mix (Thermo Scientific, California USA) containing specific oligonucleotides (0.3 micromolar; Table 1$)$ in microwells of optical 384-well plates according to manufacturer's protocol. All samples were run in triplicates. The reactions were run in the $\mathrm{ABI} \mathrm{PRISM}^{\circ} 7900 \mathrm{HT}$ sequence analyser instrument (Applied Biosystems, California, USA) with Sequence Detector System 2.2 Software following three-step cycling protocol with $95{ }^{\circ} \mathrm{C}$ for $10 \mathrm{~min}$ for initial denaturation; $95^{\circ} \mathrm{C}$ for $15 \mathrm{~s}$ to denaturation; $60^{\circ} \mathrm{C}$ of $30 \mathrm{~s}$ to elongation and $30 \mathrm{~s}$ of $72{ }^{\circ} \mathrm{C}$ to extension. After amplification, the $\mathrm{Ct}$ values were used to obtain normalized expression values as previously described [66].

\section{DNA Isolation and bisulfite treatment}

Genomic DNA samples were isolated from the hTEC line using the Wizard ${ }^{\circ}$ Genomic DNA Purification Kit (Promega, Wisconsin, USA) according to the manufacturer's instructions. After isolation, DNA concentration and purity were determined by the ratio of absorbance readings at 260 and $280 \mathrm{~nm}$ on NanoDrop ND 2000 spectrometer (Thermo Scientific, Delaware, USA). Two micrograms of DNA were used to perform bisulfite treatment using Epitect Bisulfite Kit (Qiagen, Hilden, Germany) following the supplier's instructions. After reaction, the converted DNA was purified using silica columns (EpiTect spin columns, Qiagen, Hilden, Germany), eluted in RNAse and DNAse free water and keep in -20 freezer until use.

\section{DNA Methylation analysis}

Candidate regulatory sequences for $\mathrm{CpG}$ methylation were selected from the FOXN1 gene using features of the University of California Santa Cruz (UCSC) genome browser (UCSC ID: uc010crm.3; [67]). For selection, in addition to known features relevant to probable $\mathrm{CpG}$ methylation, we used experimental data reported in UCSC genome browser, namely the DNA methylation, by reduced representation bisulfite sequencing performed by the Encyclopedia of DNA Elements (ENCODE) consortium [39]. Selected regions were exploited as targets to design bisulfite-treated DNA specific oligonucleotides using the MethylPrimer Express v 1.0 software (Applied Biosystems) and the web-based software MethPrimer [68]. Both programs have algorithms for primer design specific for bisulfite-treated DNA, according the criteria described by Li and Dahiya (2002) [68]. For bisulfite sequencing, isolated DNA from hTEC line were converted with sodium bisulfite using Epitect Bisulfite kit (Qiagen, Hilden, Germany) as described above. The selected candidate sequence (Fig. 5a and b) was amplified with specific primers for FOXN1/C20 region (F: GTTTAGGGTGAGTTAGG TAGGGATAG; R: AAAAACAAATTTCTAACTCTAAA AATAAAT). The PCR products were cloned in PGEM-T

Table 1 Forward and reverse primer sequences used on the quantitative PCR and RT-PCR assays

\begin{tabular}{llllc}
\hline Target & Sequence forward (5'-3') & Sequence reverse (5'-3') & Amplicon size (pb) & Reference \\
\hline FOXN1 (NM_003593.2) & TCCCTCACTCACTGACTTCG (1628-1647) & GTGGCATCGAAGATGATGTC (1746-1727) & 119 & [72] \\
DLL1 (NM_005618.3) & TGCAACCAGGACCTGAACTA (1323-1342) & CTCCGTCTTACAAGGGCTG (1491-1472) & 163 & $*$ \\
DLL4 (NM_019074.3) & CAGAGTGTCGGATATCAGCG (2288-2307) & CTCCTGCCTTATACCTCCGT (2402-2383) & 115 & * \\
WNT-4 (NM_030761.4) & CAGCAGAGCCCTCATGAACC (647-666) & GCCAGCACGTCTTACCTCACA (768-747) & 122 & [73] \\
HPRT-1 (NM_000194.2) & CCTGGCGTCGTGATTAGTG (183-201) & TCGAGCAAGACGTTCAGTCC (320-301) & 138 & $*$ \\
TFRC (NM_001128148.1) & CTAGTGTTCTTCTGTGTGGCAGT (115-138) & ACAATGGTTCTCCACCAAACAAG (197-175) & 83 & $*$ \\
RPL13A (NM_001270491.1) & CGGACCGTGCGAGGTATGCT (244-263) & AGCAGGAACCACCATCCGCT (366-347) & 123 & $*$ \\
\hline
\end{tabular}

HPRT-1 (hypoxanthine phosphoribosyltransferase 1), TRFC (transferrin receptor) and RPL13A (ribosomal protein L13a) were used as control housekeeping genes. (*)Designed using the software Primer3 [37]. FOXN1: forkhead box N1; DLL1: Delta-like 1 (Drosophila); DLL4: Delta-like (Drosophila) 4; WNT-4: wingless-type MMTV integration site family, member 4 . 
plasmid (pGEM ${ }^{\circ}-\mathrm{T}$ Easy Vector System I; Promega, Wisconsin, USA) and the inserts were sequenced using BigDye ${ }^{\curvearrowleft}$ Terminator Cycle Sequencing Kit (Applied Biosystems, California, USA) using forward or reverse PCR primers. The reactions were performed in ABI3730xl DNA analyzer (Applied Biosystems, California, USA) at the Fiocruz DNA sequencing platform (Rio de Janeiro, Brazil). Traces were analysed with BioEdit: Sequence Alignment Editor v. 7.2.5 (Ibis Biosciences, Califorina, USA), aligned with ClustalW v1.83 [69] and submitted to CpG quantification using $\mathrm{BiQ}$ analyzer v2.00 [70]. Percent methylation, with respect to each individual $\mathrm{CpG}$, and the global average percentage methylation per each candidate sequences were displayed. For methylation call we used $>90 \%$ conversion threshold. Lollipop diagrams were made per samples using Quantification Tool for Methylation Analysis (QUMA) web software [71].

\section{Statistical analyses}

To test the probability of significant differences among the different study groups, the data obtained were analyzed GraphPad Prism software version 5.00 (GraphPad Prism Software, Inc.) using the non-parametric one-way ANOVA Kruskal-Wallis test, followed by Dunn's post-test. The values were represented by the mean \pm standard error of the mean (SEM) and considered significant when $\mathrm{p} \leq 0.05$.

\section{Additional file}

Additional file 1 Supplementary material. (PDF 867 kb)

\begin{abstract}
Abbreviations
TCR: T cell repector; TEC: Thymic epithelial cell; ECM: Extracellular matrix; MHC: Major histocompatibility complex; FOXN1: forkhead box N1; DLL1: Delta-like 1 (Drosophila); DLL4: Delta-like (Drosophila) 4; WNT-4: winglesstype MMTV integration site family, member 4; JAG: Jagged; SV40: Simius virus 40; PAX-1: Paired box gene 1; LiCl: Lithium chloride; NaCl: Sodium chloride; ENCODE: Encyclopedia of DNA Elements; UCSC: Unversity of California Santa Cruz.
\end{abstract}

\section{Competing interests}

Authors declare that they have no competing interests.

\section{Authors' contributions}

MDS, WS and LT conceived and designed the experiments. MDS, KC and LPBD performed the experiments and analyzed the data. TS and ZP provided thymic human samples. WS and LT contributed with reagents/materials/analysis tools. MDS, WS and LT wrote the manuscript.

\section{Authors' informations \\ MDS was a CNPq/Science without Borders Program (Brazil) (Grant $n^{\circ}$ : 240788/2012-5) visiting PhD fellow at the Department of Human Genetics, Faculty of Medicine, University of Debrecen, Debrecen, Hungary. LT was a CNPq/Science without Borders Program (Brazil) (Grant n: 240788/2012-5) short time visiting research fellow at the Laboratory on Thymus Research, Oswaldo Cruz Institute, FIOCRUZ, Brazil.}

\section{Acknowledgements}

The authors acknowledge the assistance with immunofluorescence experiments, the stimulating discussions and the review of the manuscript to Istvan Ando (Department of Genetics, Biological Research Center, Szeged Hungary).
This work is support by grants from FIOCRUZ, CAPES and CNPq/Science without Borders Program (Brazil) to Maria Danielma dos Santos Reis (MDS) and Laszlo Takacs (LT) (Grant n: 240788/2012-5). The VÉD-ELEM: TÁMOP 4.2.2.A-11/1/KONV-2012-0023 grant (Hungary) supported LT's laboratory.

\section{Author details}

${ }^{1}$ Laboratory on Thymus Research, Oswaldo Cruz Institute, Oswaldo Cruz Foundation, Rio de Janeiro, Brazil. ${ }^{2}$ Department of Human Genetics, Faculty of Medicine, University of Debrecen, Nagyerdei krt. 98, H-4032 Debrecen, Hungary. ${ }^{3}$ Department of Biochemistry and Molecular Biology, Faculty of Medicine, University of Debrecen, Debrecen, Hungary. ${ }^{4}$ Gottsegen György Hungarian Institute of Cardiology, Budapest, Hungary. ${ }^{5}$ Department of Cardiac Surgery, Clinical Center, University of Debrecen, Debrecen, Hungary.

${ }^{6}$ Biosystems International Kft, Debrecen, Hungary.

Received: 15 June 2015 Accepted: 15 October 2015

Published online: 29 October 2015

\section{References}

1. Chinn IK, Blackburn CC, Manley MR, Sempowski GD. Changes in primary lymphoid organs with aging. Semin Immunol. 2012;24:309-20.

2. Farley AM, Morris LX, Vroegindeweij E, Depreter ML, Vaidya H, Stenhouse $\mathrm{FH}$, et al. Dynamics of thymus organogenesis and colonization in early human development. Development. 2013;140:2015-26.

3. Pfister G, Savino W. Can the immune system still be efficient in the elderly? An immunological and immunoendocrine therapeutic perspective. Neuroimmunomodulation. 2008;15:351-64.

4. Haynes L, Maue AC. Effects of aging on T cell function. Curr Opin Immunol. 2009;21:414-17.

5. World Health Organization. Antimicrobial resistance. Fact Sheet $n^{\circ} 194.2014$. http://www.who.int/mediacentre/factsheets/ss194/en/. Accessed 15 Feb 2015.

6. Fink PJ, Hendricks DW. Post-thymic maturation: young T cells assert their individuality. Nat Rev Immunol. 2011;11(8):544-49.

7. Savino W, Mendes-da-Cruz DA, Silva JS, Dardenne M, Cotta-de-Almeida V. Intrathymic T-cell migration: a combinatorial interplay of extracellular matrix and chemokines? Trends Immunol. 2002;23:305-13.

8. Steinmann GG, Klaus B, Muller-Hermelink HK. The involution of the ageing human thymic epithelium is independent of puberty. A morphometric study. Scand. J. Immunol. 1985;22:563-75.

9. Flores KG, Li J, Sempowski GD, Haynes BF, Hale LP. Analysis of the human thymic perivascular space during aging. J Clin Invest. 1999;104:1031-9.

10. Savino W, Dardenne M. Neuroendocrine control of thymus physiology. Endocr Rev. 2000;21:412-43.

11. Shah DK, Zúñiga-Pflücker JC. An overview of the intrathymic intricacies of T cell development. J Immunol. 2014;192:4017-23.

12. Klein L, Kyewski B, Allen PM, Hogquist KA. Positive and negative selection of the T cell repertoire: what thymocytes see (and don't see). Nat Rev Immunol. 2014;14:377-91.

13. Haynes BF, Markert ML, Sempowski GD, Patel DD, Hale LP. The role of the thymus in immune reconstitution in aging, bone marrow transplantation, and HIV-1 infection. Annu Rev Immunol. 2000;18:529-60.

14. Douek DC, McFarland RD, Keiser PH, Gage EA, Massey JM, Haynes BF, et al. Changes in thymic function with age and during the treatment of HIV infection. Nature. 1998;396:690-5.

15. Gui J, Zhu X, Dohkan J, Cheng L, Barnes PF, Su DM. The aged thymus shows normal recruitment of lymphohematopoietic progenitors but has defects in thymic epithelial cells. Int Immunol. 2007;19:1201-11.

16. Aw D, Silva AB, Maddick M, von Zglinicki T, Palmer DB. Architectural changes in the thymus of aging mice. Aging Cell. 2008;7:158-67.

17. Su DM, Navarre S, Oh WJ, Condie BG, Manley NR. A domain of Foxn1 required for crosstalk-dependent thymic epithelial cell differentiation. Nat Immunol. 2003:4:1128-35.

18. Bredenkamp N, Ulyanchenko S, O'Neill KE, Manley NR, Vaidya HJ, Blackburn CC. An organized and functional thymus generated from FOXN1-reprogrammed fibroblasts. Nat Cell Biol. 2014;16:902-8.

19. Balciunaite G, Keller MP, Balciunaite E, Piali L, Zuklys S, Mathieu YD, et al. Wnt glycoproteins regulate the expression of FoxN1, the gene defective in nude mice. Nat Immunol. 2002;3(11):1102-8.

20. Blackburn CC, Manley NR. Developing a new paradigm for thymus organogenesis. Nat Rev Immunol. 2004;4(4):278-89. 
21. Nowell CS, Bredenkamp N, Tetélin S, Jin X, Tischner C, Vaidya H, et al. Foxn1 regulates lineage progression in cortical and medullary thymic epithelial cells but is dispensable for medullary sublineage divergence. PLOS Genet. 2011;7(11), e1002348.

22. Boehm T. Thymus development and function. Curr Opin Immunol. 2008;20(2):178-84.

23. Nehls M, Pfeifer D, Schorpp M, Hedrich H, Boehm T. New member of the winged-helix protein family disrupted in mouse and rat nude mutations. Nature. 1994;372(6501):103-7.

24. Schüddekopf $K$, Schorpp M, Boehm T. The whn transcription factor encoded by the nude locus contains an evolutionarily conserved and functionally indispensable activation domain. Proc Natl Acad Sci U S A. 1996;93(18):9661-4.

25. Frank J, Pignata C, Panteleyev AA, Prowse DM, Baden $H$, Weiner $L$, et al. Exposing the human nude phenotype. Nature. 1999;398(6727):473-4.

26. Ortman $\mathrm{CL}$, Dittmar KA, Witte PL, Le PT. Molecular characterization of the mouse involuted thymus: aberrations in expression of transcription regulators in thymocyte and epithelial compartments. Int Immunol. 2002;14(7):813-22.

27. Kvell K, Varecza Z, Bartis D, Hesse S, Parnell S, Anderson G, et al. Wnt4 and LAP2alpha as pacemakers of thymic epithelial senescence. PLoS One. 2010;5(5), e10701.

28. Chen L, Xiao S, Manley NR. Foxn1 is required to maintain the postnatal thymic microenvironment in a dosage-sensitive manner. Blood. 2009;113(3):567-74.

29. Sun L, Guo J, Brown R, Amagai T, Zhao Y, Su DM. Declining expression of a single epithelial cell-autonomous gene accelerates age-related thymic involution. Aging Cell. 2010;9(3):347-57.

30. Zook EC, Krishack PA, Zhang S, Zeleznik-Le NJ, Firulli AB, Witte PL, et al. Overexpression of Foxn1 attenuates age-associated thymic involution and prevents the expansion of peripheral CD4 memory T cells. Blood. 2011;118(22):5723-31.

31. Bredenkamp N, Nowell CS, Blackburn CC. Regeneration of the aged thymus by a single transcription factor. Development. 2014;141:1627-37.

32. Romano R, Palamaro L, Fusco A, lannace L, Maio S, Vigliano I, et al. From murine to human nude/SCID: the thymus, T-cell development and the missing link. Clin Dev Immunol. 2012;467101.

33. Fernández E, Vicente A, Zapata A, Brera B, Lozano JJ, Martínez C, et al. Establishment and characterization of cloned human thymic epithelial cell lines. Analysis of adhesion molecule expression and cytokine production. Blood. 1994;83(11):3245-54.

34. Lamberts SW, Van Den Beld AW, Van Der Lely AJ. The endocrinology of aging. Science. 1997;278(337):419-24.

35. Anderson KL, Moore NC, McLoughlin DE, Jenkinson EJ, Owen JJ. Studies on thymic epithelial cells in vitro. Dev Comp Immunol. 1998;22:367-77.

36. Beaudette-Zlatanova BC, Knight KL, Zhang S, Stiff PJ, Zúñiga-Pflücker JC, Le PT. A human thymic epithelial cell culture system for the promotion of lymphopoiesis from hematopoietic stem cells. Exp Hematol. 2011;39:570-9.

37. Mohtashami M, Zúñiga-Pflücker JC. Three-dimensional architecture of the thymus is required to maintain delta-like expression necessary for inducing T cell development. J Immunol. 2006;176:730-4.

38. Stambolic V, Ruel L, Woodgett JR. Lithium inhibits glycogen synthase kinase-3 activity and mimics wingless signalling in intact cells. Curr Biol. 1996;6:1664-8

39. ENCODE project consortium. An integrated encyclopedia of DNA elements in the human genome. Nature. 2012;489(7414):57-74.

40. Mandinova A, Kolev V, Neel V, Hu B, Stonely W, Lieb J, et al. A positive FGFR3/ FOXN1 feedback loop underlies benign skin keratosis versus squamous cell carcinoma formation in humans. J Clin Invest. 2009:119(10):3127-37.

41. Su Al, Wiltshire T, Batalov S, Lapp H, Ching KA, Block D, et al. A gene atlas of the mouse and human protein-encoding transcriptomes. Proc Natl Acad Sci U S A. 2004;101(16):6062-7.

42. Plowden J, Renshaw-Hoelscher M, Engleman C, Katz J, Sambhara S. Innate immunity in aging: impact on macrophage function. Aging Cell. 2004;3(4):161-7.

43. Fink PJ. The biology of recent thymic emigrants. Annu Rev Immunol. 2013;31:31-50

44. Kilpatrick RD, Rickabaugh T, Hultin LE, Hultin P, Hausner MA, Detels R, et al. Homeostasis of the naive CD4+ T cell compartment during aging. J Immunol. 2008;180(3):1499-507.

45. den Braber I, Mugwagwa T, Vrisekoop N, Westera L, Mögling R, de Boer AB, et al. Maintenance of peripheral naive $T$ cells is sustained by thymus output in mice but not humans. Immunity. 2012;36(2):288-97.
46. Boehm T, Swann JB. Thymus involution and regeneration: two sides of the same coin? Nat Rev Immunol. 2013;13:831-8.

47. Koch U, Fiorini E, Benedito R, Besseyrias V, Schuster-Gossler K, Pierres M, et al. Delta-like 4 is the essential, non redundant ligand for Notch1 during thymic T cell lineage commitment. J Exp Med. 2008;205(11):2515-23.

48. Louis I, Heinonen KM, Chagraoui J, Vainio S, Sauvageau G, Perreault C. The signaling protein Wnt4 enhances thymopoiesis and expands multipotent hematopoietic progenitors through beta-catenin-independent signaling. Immunity. 2008;29(1):57-67.

49. Felli MP, Maroder M, Mitsiadis TA, Campese AF, Bellavia D, Vacca A, et al. Expression pattern of notch 1, 2 and 3 and Jagged 1 and 2 in lymphoid and stromal thymus components: distinct ligand-receptor interactions in intrathymic T cell development. Int Immunol. 1999;11(7):1017-25.

50. Fiorini E, Ferrero I, Merck E, Favre S, Pierres M, Luther SA, et al. Cutting edge: thymic crosstalk regulates delta-like 4 expression on cortical epithelial cells. J Immunol. 2008;181(12):8199-203.

51. Van de Walle I, De Smet G, Gärtner M, De Smedt M, Waegemans E, Vandekerckhove B, et al. Jagged 2 acts as a Delta-like Notch ligand during early hematopoietic cell fate decisions. Blood. 2011;117(17):4449-59.

52. Hozumi K, Mailhos C, Negishi N, Hirano K, Yahata T, Ando K, et al. Delta-like 4 is indispensable in thymic environment specific for T cell development. J Exp Med. 2008;205(11):2507-13.

53. Weerkamp F, Baert MRM, Naber BAE, Koster EEL, de Haas EFE, Atkuri KR, et al. Wnt signaling in the thymus is regulated by differential expression of intracellular signaling molecules. Proc Natl Acad Sci U S A. 2006;103:3322-6.

54. Pongracz J, Hare K, Harman B, Anderson G, Jenkinson EJ. Thymic epithelial cells provide WNT signals to developing thymocytes. Eur J Immunol. 2003;33(7):1949-56.

55. Osada M, Jardine L, Misir R, Andl T, Millar SE, Pezzano M. DKK1 mediated inhibition of Wnt signaling in postnatal mice leads to loss of TEC progenitors and thymic degeneration. PLoS One. 2010;5(2), e9062.

56. Fukuda D, Aikawa E, Swirski FK, Novobrantseva TI, Kotelianski V, Gorgun CZ, et al. Notch ligand delta-like 4 blockade attenuates atherosclerosis and metabolic disorders. Proc Natl Acad Sci U S A. 2012;109(27):E1868-77.

57. Pignata C, Fiore M, Guzzetta V, Castaldo A, Sebastio G, Porta F, et al. Congenital Alopecia and nail dystrophy associated with severe functional T-cell immunodeficiency in two sibs. Am J Med Genet. 1996;65(2):167-70.

58. Schubeler D. Function and information content of DNA methylation. Nature. 2015;517:321-6.

59. Romanov GA, Vanyushin BF. Methylation of reiterated sequences in mammalian DNAs. Effects of the tissue type, age, malignancy and hormonal induction. Biochim Biophys Acta. 1981;653:204-18.

60. Wilson VL, Smith RA, Ma S, Cutler RG. Genomic 5-methyldeoxycytidine decreases with age. J Biol Chem. 1987;262(21):9948-51.

61. Pogribny IP, Vanyushin BF. Age-Related Genomic Hypomethylation. In: Tollefsbol TO, editor. Epigenetics of Aging. New York: Springer; 2010. p. 11-27.

62. Golbert DC, Correa-de-Santana E, Ribeiro-Alves M, de Vasconcelos AT, Savino W. ITGA6 gene silencing by RNA interference modulates the expression of a large number of cell migration-related genes in human thymic epithelial cells. BMC Genomics. 2013;14 Suppl 6:S3.

63. Paraguassú-Braga FH, Alves AP, Santos IM, Bonamino M, Bonomo A. An ectopic stromal implant model for hematopoietic reconstitution and in vivo evaluation of bone marrow niches. Cell Transplant. 2012;21(12):2677-88.

64. Staal FJ, Weerkamp F, Baert MR, van den Burg CM, van Noort M, de Haas EF, et al. Wht target genes identified by DNA microarrays in immature CD34+ thymocytes regulate proliferation and cell adhesion. J Immunol. 2004;172(2):1099-108.

65. Ye J, Coulouris G, Zaretskaya I, Cutcutache I, Rozen S, Madden TL. PrimerBLAST: a tool to design target-specific primers for polymerase chain reaction. BMC Bioinformatics. 2012;13:134.

66. Vandesompele J, De Preter K, Pattyn F, Poppe B, Van Roy N, De Paepe A, et al. Accurate normalization of real-time quantitative RT-PCR data by geometric averaging of multiple internal control genes. Genome Biol. 2002;3(7):RESEARCH0034.

67. Kent WJ, Sugnet CW, Furey TS, Roskin KM, Pringle TH, Zahler AM, et al. The Human Genome Browser at UCSC. Genome Res. 2002;12(6):996-1006.

68. Li LC, Dahiya R. MethPrimer: designing primers for methylation PCRs. Bioinformatics. 2002;18:1427-31.

69. Larkin MA, Blackshields G, Brown NP, Chenna R, McGettigan PA, McWilliam H, et al. Clustal $W$ and Clustal X version 2.0. Bioinformatics. 2007;23(21):2947-8. 
70. Bock C, Reither S, Mikeska T, Paulsen M, Walter J, Lengauer T. BiQ Analyzer: visualization and quality control for DNA methylation data from bisulfite sequencing. Bioinformatics. 2005;21(21):4067-8.

71. Kumaki Y, Oda M, Okano M. QUMA: quantification tool for methylation analysis. Nucleic Acids Res. 2008;36(Web Server issue):W170-5.

72. Clark RA, Yamanaka K, Bai M, Dowgiert R, Kupper TS. Human skin cells support thymus-independent T cell development. J Clin Invest. 2005:115(11):3239-49.

73. Liu J, Wang Y, Pan Q, Su Y, Zhang Z, Han J, et al. Wnt/ $\beta$-catenin pathway forms a negative feedback loop during TGF- $\beta 1$ induced human normal skin fibroblast-to-myofibroblast transition. J Dermatol Sci. 2012;65(1):38-49.

74. Smit A, Hubley R, Green P. RepeatMasker Open-4.0. 2013-2015. http://www.repeatmasker.org. Accessed 23 Dec 2014

\section{Submit your next manuscript to BioMed Central and take full advantage of:}

- Convenient online submission

- Thorough peer review

- No space constraints or color figure charges

- Immediate publication on acceptance

- Inclusion in PubMed, CAS, Scopus and Google Scholar

- Research which is freely available for redistribution 